\title{
Mechanistic Links between Non-Coding RNAs and Myeloid Cell Inflammation in Atherosclerosis
}

\author{
Valentina Paloschi ${ }^{1,2, *(1)}$ \\ Hanna Winter ${ }^{1,2, *}$ \\ ${ }^{1}$ Department of Vascular and Endovascular Surgery, Technical \\ University of Munich, Munich, Germany \\ ${ }^{2}$ German Center for Cardiovascular Research, Partner Site Munich, \\ Munich, Germany \\ 3 Institute for Prevention and Epidemiology of Cardiovascular \\ Diseases, Ludwig-Maximilians-University Munich, Munich, Germany \\ ${ }^{4}$ Department of Physiology and Pharmacology, Karolinska Institute, \\ Stockholm, Sweden \\ ${ }^{5}$ Department of Medicine, Karolinska Institute, Stockholm, Sweden
}

Joana Viola ${ }^{2,3}$

Oliver Soehnlein $2,3,4$

Lars Maegdefessel $\mathrm{l}^{1,2,5}$

\author{
Address for correspondence Valentina Paloschi, PhD, Vascular Biology \\ Unit, Department of Vascular and Endovascular Surgery, Klinikum rechts \\ der Isar - Technical University of Munich, Biedersteiner Strasse 29, \\ 80802 Munich, Germany (e-mail: valentina.paloschi@tum.de).
}

Thromb Haemost 2019;119:1205-1211.

\begin{abstract}
Keywords

- atherosclerosis

- non-coding RNAs

- vascular inflammation

Inflammation plays a pivotal role in the chronicity of atherosclerotic lesion development and progression. Myeloid cells are involved in all stages of atherosclerosis development: they contribute in early phases to endothelial dysfunction and create a pro-inflammatory environment responsible for disease progression. Numerous studies over the last decade have repeatedly provided evidence for the crucial importance for different classes of non-coding ribonucleic acids (RNAs) in regulating gene expression, as well as messenger RNA and protein stability. Functional studies using tools to either over-express or inhibit these non-coding RNAs showcased strong effects on tempering vascular inflammation and atherosclerosis progression. With this current review article, we want to discuss prominent examples of non-coding RNAs, being either produced by myeloid cells or affecting their recruitment and activity in the context of vascular inflammation, atherosclerosis and consequential diseases (such as myocardial infarction and stroke). All of the discussed transcripts were thoroughly studied in mechanistic explorations, indicating that they have the capability to modulate inflammatory cascades in the vasculature during disease exacerbation.
\end{abstract}

\section{Myeloid Cell-Driven Vascular Inflammation during Atherosclerosis Progression: Focus on Non-Coding Ribonucleic Acid}

Atherosclerosis is defined as a chronic disease of the arterial vessel wall whose architecture is slowly remodelled during time. The endothelial layer, at the interface with circulating

\footnotetext{
* Valentina Paloschi and Hanna Winter equally contributed to the study.

(1) Valentina Paloschi's ORCID is https://orcid.org/0000-0002-57788981.
}

blood cells, plays a relevant role in the initial stage of atherosclerosis. Subsequent pathological remodelling of the medial layer populated by smooth muscle cells (SMCs) leads to lesion formation and evolution into an atherosclerotic plaque. In the present review, we discuss mechanisms responsible for the chronicity of atherosclerotic disease, with a focus on myeloid cells contribution in aggravating the inflammatory status during lesion progression and destabilisation.

Myeloid cells originate from a common myeloid progenitor during haematopoiesis. They include thrombocytes, erythrocytes, mast cells and myeloblast-derived cells that are basophils, neutrophils, eosinophils and monocytes. Among those, the

(c) 2019 Georg Thieme Verlag KG Stuttgart · New York
DOI https://doi.org/ 10.1055/s-0039-1687874. ISSN 0340-6245.
September 28, 2018

accepted after revision

February 27, 2019 
monocytes play a predominant role in the initiation and also in the advancement of atherosclerosis. Monocytes can differentiate into macrophages and myeloid lineage dendritic cells (DCs), thereby triggering and perpetuating inflammatory processes. DCs of myeloid origin are sub-populations of monocytes/ macrophages, potent activators of the adaptive immune system, also known as antigen presenting cells.

The contribution of neutrophils, which are the most abundant leucocyte sub-type in human circulation, is mostly described in acute inflammatory responses whereas their contribution to chronic inflammation has only recently been appreciated. Neutrophils are found in both human and murine atherosclerotic plaques throughout different stages of the disease ${ }^{1,2}$ underscoring their contribution to this chronic form of inflammation. In fact, recent studies have shed light on the importance of neutrophils during atherosclerosis and its complications. ${ }^{1,3,4}$

In the present review, we provide examples of how, 'molecular messages' originating from myeloid cells can be detrimental for vascular wall cells with the formation of advanced atherosclerotic lesions as a consequence. As for 'molecular messages', we focus on non-coding ribonucleic acids (RNAs), for their potential to orchestrate pathological changes occurring during disease.

Previously, it was assumed that roughly $98 \%$ of the genome is 'junk deoxyribonucleic acid (DNA)/RNA'. Through next-generation sequencing and other novel RNA/DNA profiling techniques, it has been shown that non-coding RNAs possess key relevance in the regulation of gene expression. This current review focuses on better-studied micro-RNAs (miRNAs) and long non-coding RNAs (lncRNAs) and their capability to function as a means of intercellular communication between myeloid cells and resident cells of the vessel wall, such as endothelial cells (ECs) and SMCs, in the context of atherosclerosis ( $\mathbf{- T a b l e ~} \mathbf{1}$ ).

MiRNAs are small (21-23 nucleotides [nt]), non-coding RNAs that regulate gene expression on the post-transcriptional level. ${ }^{5}$ One processed strand gets loaded into the miRNA-induced silencing complex, where it interacts with the Argonaute protein to bind to the 3 ' untranslated region of the target sequence. Thus, messenger RNA (mRNA) translation within this target sequence is inhibited. ${ }^{5}$ By this function, miRNAs were discovered to take part in several important biological processes and-following-also in disease development by orchestrating gene expression via inhibiting crucial target mRNAs. Therefore, they are considered as key mediators that can be used as biomarker or even as a potential therapeutic target.

LncRNAs, which by definition are longer than $200 \mathrm{nt}$, are less well characterised and conserved compared with miRNAs but more tissue-specific. Their function also differs from miRNAs, as they can share complementary exons with their targets, which can be found in both sense and anti-sense direction of an lncRNA. ${ }^{6}$ They can function as a scaffold for transcription factors (lincRNAs), enhancers (eRNAs) or as sponges for miRNAs. ${ }^{7}$ Thus, IncRNAs are able to communicate/interact with both proteins and also DNA or RNAs. Even though they appear to be distinctively different from
miRNAs, their ultimate purpose is to orchestrate cellular processes and to filter unnecessary 'genetic/molecular noise'. ${ }^{6}$ One prominent example of a lncRNA in atherosclerotic diseases is certainly cyclin-dependent kinase inhibitor 2B-antisense 1 or ANRIL, which was identified in a chromosomal region (9p21.3) strongly linked to cardiovascular disease. ${ }^{8}$ Although the molecular mechanisms of how ANRIL and its numerous isoforms are regulated by the genotype at the locus have not fully been elucidated, its presence in circulating blood and within the atherosclerotic tissue has been showed by several, highlighting its crucial role as intercellular mediator in atherosclerosis. ${ }^{9-11}$

\section{Relevant Non-Coding RNAs Regulating Vascular Inflammation: Myeloid Cell-Borne Non-Coding RNAs}

During atherogenesis, monocytes infiltrate the vessel wall, undergo transition to macrophages, which then start engulfing lipids, and activate inflammatory signals. Macrophages also regulate cholesterol efflux, via adenosine triphosphate (ATP)-binding cassette (ABC) transporters, which are important contributors to atherosclerotic plaque development and progression. MiR-21 is the most abundantly expressed miRNA in macrophages, and has been shown to negatively regulate pro-inflammatory responses. ${ }^{12}$ Its absence in macrophages on the contrary accelerates vascular inflammation, by enhancing ATP binding cassette subfamily G member 1 , and by this augmenting the number of macrophages that turn into foam cells. ${ }^{12}$

MiRNA-146a plays an important role in monocyte and macrophage activation by negatively regulating nuclear factor-kappa B (NF-kB), if abundantly expressed in these cell types. ${ }^{13}$ This activity was found to be influenced and steered by apolipoprotein $\mathrm{E}$ (ApoE) by enhancing the transcription factor PU.1. ${ }^{13}$ Delivery of miR-146a mimics to Apo $E^{-/-}$Ldlr $^{-/-}$ (low-density lipoprotein receptor) mice led to alleviation of inflammation-related symptoms, such as a reduced number of blood monocytes, as well as down-regulated expression of NF-KB and tumour necrosis factor $\alpha .{ }^{13}$ MiR-146 deficient $\mathrm{Ldlr}^{-1-}$ mice, however, show increased levels of circulating leukocytes (neutrophils and monocytes) during early stages, followed by a decrease due to bone marrow failure with an overall protective effect for the animals which appear less susceptible to develop atherosclerosis. ${ }^{14}$

Further, it has been indicated that miRNA-146a is transferred from atherosclerotic macrophages to naive macrophages via extracellular vesicles to halt migration by repressing the insulin-like growth factor 2 mRNA binding protein 1 and human antigen $R$, which are both key players in migration by modulating $\beta$-actin expression. ${ }^{15}$

Interestingly, some mRNAs can also function in two different ways depending on the stage of the disease. For example, in early atherosclerosis miR-155 inhibits macrophage proliferation/influx by repressing colony stimulating factor (CSF) receptor 1 that normally reacts to external CSF with preventing lesion formation. ${ }^{16}$ In advanced atherosclerosis, miR-155 however targets B-cell lymphoma 6, 
Table1 Non-coding RNAs regulation of vascular inflammation

\begin{tabular}{|c|c|c|c|c|}
\hline ncRNA & Model organism & Modulation and target & Experimentally validated function & Ref \\
\hline miR-10a & Mouse; Human EC & $\uparrow$ to inhibit NF-kB pathway via IRAK4 & Inhibits monocytes attachment & 42 \\
\hline miR-21 & $\begin{array}{l}\text { Mouse; Mouse macrophages } \\
\text { (peritoneal, bone marrow-derived) }\end{array}$ & $\uparrow$ to inhibit JNK signalling via ABCG1 & $\begin{array}{l}\text { Regulates foam cells } \\
\text { transformation }\end{array}$ & 10 \\
\hline miR-92a & $\begin{array}{l}\text { Human ECs, macrophages and } \\
\text { SMC; bovine aortic EC }\end{array}$ & $\begin{array}{l}\downarrow \text { to prevent inhibition of KLF2, } \\
\text { KLF4 }\end{array}$ & $\begin{array}{l}\text { Regulates EC activation by oxLDL } \\
\text { under low shear stress }\end{array}$ & $\begin{array}{l}14-16 \\
\end{array}$ \\
\hline miR-126 & Mouse; Human EC and SMC & $\uparrow$ to inhibit VCAM-1, CXCL12 & $\begin{array}{l}\text { Regulates leukocyte trafficking to } \\
\text { sites of inflammation }\end{array}$ & 40,41 \\
\hline miR-142 & Mouse; Mouse stromal cells & $\downarrow$ to induce STAT1a, IRF1b & Reduces neutrophil count & 28 \\
\hline miR-146a & $\begin{array}{l}\text { Mouse; Mouse aortic cells, mono- } \\
\text { cytes and macrophages }\end{array}$ & $\uparrow$ to inhibit NF-KB via IRAK1, TRAF6 & $\begin{array}{l}\text { Reduces monocytes-derived } \\
\text { inflammation }\end{array}$ & 11 \\
\hline miR-155 & Mouse; Mouse macrophages & $\begin{array}{l}\uparrow \text { to inhibit CSFR1, } \downarrow \text { to activate } \\
\text { BCL6 }\end{array}$ & $\begin{array}{l}\text { Suppresses macrophage } \\
\text { proliferation; impairs efferocytosis }\end{array}$ & 13 \\
\hline miR-181a & Rat aortic SMC; Mouse SMC & $\uparrow$ to inhibit OPN expression & $\begin{array}{l}\text { Induces phenotypic switch in } \\
\text { smooth muscle cells }\end{array}$ & 39 \\
\hline miR-181a & Mouse; Mouse dendritic cells & $\uparrow$ to inhibit cFOS & $\begin{array}{l}\text { Activates anti-oxLDL-stimulated } \\
\text { immune inflammation responses }\end{array}$ & 27 \\
\hline miR-181b & Mouse; Human monocytes, EC & $\uparrow$ to inhibit importin- $\alpha 3$ & $\begin{array}{l}\text { Reduces NF-kB inflammatory } \\
\text { pathway cascade }\end{array}$ & 37 \\
\hline miR-195 & $\begin{array}{l}\text { Human monocytes, SMC, } \\
\text { macrophages }\end{array}$ & $\uparrow$ to inhibit IL-1 $\beta$, IL-6, TNF- $\alpha$ & $\begin{array}{l}\text { Induces polarisation towards } \\
\text { M2-anti-inflammatory phenotype }\end{array}$ & 22 \\
\hline miR-223 & Human EC and platelets & $\downarrow$ to prevent inhibition of IGF1R & $\begin{array}{l}\text { Induces apoptosis of vascular } \\
\text { endothelial cells }\end{array}$ & 34 \\
\hline miR-223 & Mouse; Mouse granulocytes & $\uparrow$ to inhibit MEF2C & $\begin{array}{l}\text { Regulates expansion and activity of } \\
\text { neutrophils }\end{array}$ & 31 \\
\hline miR-223 & $\begin{array}{l}\text { Mouse; Mouse macrophages and } \\
\text { dendritic cells }\end{array}$ & $\uparrow$ to inhibit NLRP3 & $\begin{array}{l}\text { Regulates neutrophil-derived } \\
\text { inflammation }\end{array}$ & 32 \\
\hline miR-223 & Mouse; Human neutrophils & $\uparrow$ to inhibit PARP-1 & Dampens acute lung inflammation & 35 \\
\hline ANRIL & $\begin{array}{l}\text { Human vascular tissue and } \\
\text { peripheral blood }\end{array}$ & $\downarrow$ to prevent inhibition of CDKN2B/A & $\begin{array}{l}\text { Associated to the cardiovascular } \\
\text { disease locus 9p21.3 }\end{array}$ & 8 \\
\hline HOTAIR & Human macrophages & $\begin{array}{l}\downarrow \text { to prevent inhibition of ABCA1, } \\
\text { miR-330-5p }\end{array}$ & Influences cholesterol efflux & 19 \\
\hline LINC00305 & Human monocytes and SMC & $\begin{array}{l}\downarrow \text { to prevent inhibition of LIMR, } \\
\text { AHRR }\end{array}$ & $\begin{array}{l}\text { Increases inflammatory genes and } \\
\text { drives SMC synthetic phenotype }\end{array}$ & 23,24 \\
\hline GAS5 & $\begin{array}{l}\text { Human monocytes, macrophages } \\
\text { and EC }\end{array}$ & $\downarrow$ to inhibit apoptosis pathway & $\begin{array}{l}\text { Induces apoptosis of vascular } \\
\text { endothelial cells }\end{array}$ & 36 \\
\hline MALAT1 & $\begin{array}{l}\text { Mouse; Human monocytes, } \\
\text { macrophages }\end{array}$ & $\begin{array}{l}\uparrow \text { to induce interaction with } \\
\text { IncNEAT }\end{array}$ & $\begin{array}{l}\text { A deficiency leads to immune system } \\
\text { dysregulation and atherosclerosis }\end{array}$ & 20 \\
\hline MEXIS & Mouse; Mouse macrophages & $\begin{array}{l}\uparrow \text { to activate LXR-dependent gene } \\
\text { via DDX17 }\end{array}$ & $\begin{array}{l}\text { Modulates inflammatory cytokines } \\
\text { production }\end{array}$ & 18 \\
\hline RNCR3 & Mouse; Human EC and SMC & $\begin{array}{l}\uparrow \text { to form a feedback loop with KLF2 } \\
\text { and miR-185-5p }\end{array}$ & $\begin{array}{l}\text { Protects against hypercholestero- } \\
\text { lemia-induced EC and SMC } \\
\text { dysfunction }\end{array}$ & 43 \\
\hline
\end{tabular}

Abbreviations: ABCG1, ATP binding cassette subfamily G member 1; AHRR, aryl hydrocarbon receptor repressor; ATP, adenosine triphosphate; BCL6, B-cell lymphoma 6; CDKN2B, cyclin-dependent kinase inhibitor 2B; CSFR1, colony stimulating factor receptor 1; CXCL12, C-X-C motif chemokine ligand 12; EC, endothelial cell; IGF1R, insulin-like growth factor 1 receptor; IL-1, interleukin 1; IRAK4, IL-1 receptor associated kinase 4; IRF1b, interferon regulatory factor 1 ; JNK, c-Jun N-terminal kinase; KLF2, Krüppel-like factor 2; LIMR, Lipocalin-1 interacting membrane receptor; LXR, liver X receptor; MEF2C, myocyte enhancer factor-2C; NF-KB, nuclear factor-kappa B; NLRP3, NLR family pyrin domain containing 3; OPN, osteopontin; oxLDL, oxidised low-density lipoprotein; PARP-1, poly(adenosine diphosphate-ribose) polymerase-1; RNA, ribonucleic acid; SMC, smooth muscle cell; STAT1a, signal transducer and activator of transcription 1; TNF- $\alpha$, tumour necrosis factor $\alpha$; TRAF6, TNF receptor-associated factor 6; VCAM-1, vascular cell adhesion molecule 1.

which leads to impaired efferocytosis further fuelling the process of unstable lesion formation. ${ }^{16}$

Loyer et al were able to show that miR-92a up-regulation correlated with low shear stress as well as elevated oxidised LDL (oxLDL) levels. ${ }^{17}$ By utilising miR-92a antagomirs (inhi- bitors), smaller plaque size as well as a more stable phenotype could be achieved in vivo. ${ }^{17}$ Further, over-expression of miR-92a was shown to lower expression levels of Krüppellike factors $2^{18}$ and $4^{19}$, which could be reversed by depleting miR-92a via using site-specific inhibitors. ${ }^{18}$ 
The therapeutic potential of anti-mir92a was originally discovered in ischaemic diseases by preventing down-regulation of several pro-angiogenic proteins. ${ }^{20}$ Mir92a can also target autophagy and metabolism-related genes in a cellspecific manner. Its inhibition exerts beneficial effects also in the case of post-infarction events as highlighted by a recent publication. ${ }^{21}$ Here, anti-mir92a was shown to induce autophagy in ECs, which confers resistance to hypoxia and nutrient stress response post-infarction by providing access to energy substrates.

Another recent study performed in mouse macrophages highlighted a long non-coding RNA with a human homologue: macrophage-expressed liver $\mathrm{X}$ receptor-induced sequence (MEXIS).22 Loss of MEXIS impairs the cellular response to cholesterol overload by preventing ATP binding cassette subfamily A member 1 transcription, and accelerates inflammatory mechanisms leading to atherosclerosis. ${ }^{22}$ Long non-coding RNA homeobox transcript anti-sense RNA (HOTAIR) was also discovered for its role in vascular inflammation. When macrophages encounter oxLDL, HOTAIR is upregulated and modulates the inflammatory cytokine production. ${ }^{23}$ Furthermore, the long non-coding RNA metastasis associated lung adenocarcinoma transcript 1 (MALAT1) has been linked to immune system-mediated atherosclerosis in mice. ${ }^{10}$ Loss of MALAT1 in monocyte-derived macrophages amplifies the inflammatory responses, which can then aggravate atherosclerosis development. ${ }^{24}$

In addition, resident macrophages in atherosclerotic lesions can respond to various micro-environmental stimuli and modify their functional phenotypes. ${ }^{25}$ Mir-195 was shown to regulate macrophage polarisation towards the M2 antiinflammatory phenotype by targeting the autocrine Toll-like receptor 2 inflammatory signalling pathway. ${ }^{12}$ In pro-inflammatory M1 macrophages, mir-195 is decreased, and an artificial induction was able to prevent migration of the underlying co-cultured SMCs. ${ }^{26}$ This work further confirms the role of non-coding RNAs in mediating effects among different cells type by acting as powerful paracrine signals.

It has further been reported that monocytes express the long intergenic non-coding RNA 00305 (LINC00305), a lincRNA encoded from a region that contains single-nucleotide polymorphisms reported in genome-wide association study databases of atherosclerosis. ${ }^{27}$ Increased levels of LINC00305 in primary monocytes activated NF-KB signalling pathways, and contributed to increased expression of inflammation-associated genes. ${ }^{13}$ A secondary effect is exerted on SMCs, co-cultured with monocytes to mimic the atherosclerotic lesion microenvironment. Here, SMCs shift towards a synthetic phenotype, and thus contribute to disease progression. Furthermore, LINC00305 was shown to affect apoptosis of ECs via sponging miR-136. ${ }^{28}$

DCs share with macrophages a common myeloid progenitor termed macrophage-DC progenitor. ${ }^{29}$ The presence of inflamed lipids, such as oxLDL, leads to maturation and accumulation of DCs in the arterial wall, an event that amplifies local inflammatory processes and leads to an advanced atherosclerotic plaque phenotype, including extracellular matrix (ECM) degradation and necrotic core devel- opment. ${ }^{30}$ It was found that miR-181a is up-regulated in DCs exposed to oxLDL. By reducing c-Fos protein expression, miR181 activates an anti-inflammatory program, suggesting that non-coding RNAs can substantially trigger myeloid inflammation in vascular diseases. ${ }^{31}$

Since neutrophils are known to have a short lifespan, the importance of non-coding RNAs has been partially neglected. The relevance of miRNAs in neutrophils can, however, be inferred from a CCAAT enhancer-binding protein alpha $(\mathrm{C} / \mathrm{EBP} \alpha)$-Cre-driven Dicer1 deletion mouse line. The $C / E B P \alpha$ promoter increases its activity during the transition from common myeloid progenitors to granulocyte and monocyte progenitors. Dicer deletion in these progenitor cells causes myeloid dysplasia and decreases neutrophils in peripheral blood. ${ }^{32}$ Until now, several of these small finetuners of gene expression have been reported to be involved in neutrophil biology. ${ }^{33,34}$

Both miRs-142 and -223 have been particularly studied in the context of neutrophils and vascular inflammation. In mice, miR-142 expression is restricted to haematopoietic tissues. $^{35}$ Murine depletion of miR-142a/b show reduced numbers of both neutrophils and macrophages during foetal myelopoiesis, with neutrophils being aberrantly scattered around the head. ${ }^{36}$ Transcriptomic studies suggest that the increased expression of the genes signal transducer and activator of transcription 1 and interferon regulatory factor 1 might mediate the observed changes on the neutrophil population and morphology seen in the miR-142-1- mice. ${ }^{37}$ MiR-223 is highly expressed in myeloid progenitor cells, and is particularly increased in granulocytes. ${ }^{35}$ Via a transcriptional factor that promotes myeloid progenitor proliferation (MEF2c), miR-223 negatively regulates the expansion and activity of neutrophils. ${ }^{38}$ Neutrophil-driven inflammation is also regulated by miR-223, with one possible mechanism being the targeting of the NLR family pyrin domain containing 3 inflammasome, suppressing its activity and hereby decreasing interleukin 1 beta (IL-1 $\beta$ ) secretion. ${ }^{39}$

Intercellular communication is performed by microparticles, which can be generated from many cell types, via transfer of proteins and RNAs to target cells. ${ }^{40}$ MiR-223 is an interesting intercellular mediator found in microvesicles released by activated circulating thrombocytes, and expressed at elevated levels in atherosclerotic patients. Thrombocyte-shed miR-223 is capable of triggering apoptosis in ECs upon uptake. ${ }^{41}$ Interestingly, neutrophil-derived miR-223 can also dampen acute lung inflammation upon intercellular transfer from neutrophils to epithelial cells, possibly by repressing poly(adenosine diphosphate-ribose) polymerase- 1 in epithelial cells. ${ }^{42}$ Whether neutrophilderived particles that carry miR-223 may play similar roles in cardiovascular diseases remains yet to be addressed.

An example of another non-coding RNA being transferred in exosomes from macrophages that populate the arterial wall during lesion progression is the lncRNA growth arrest specific 5 (GAS5). ${ }^{43}$ LncRNA GAS5 has a detrimental effect on vascular ECs that receive a 'death message' from circulating monocytes, which can further infiltrate the damaged endothelium, thereby promoting vascular inflammation. 


\section{Relevant Non-Coding RNAs Regulating Vascular Inflammation: Vascular Wall-Borne Non-Coding RNAs}

As an alternative to myeloid-borne non-coding RNAs, controlling critical aspects of the vascular cells homeostasis, the vascular cells themselves can respond to the inflammatory environment, characteristic of advanced lesions, by impairing non-coding RNA expression profiles. MiR-181b was found to be decreased in ECs exposed to inflammatory stimuli with consequent activation of NF-KB signalling, suggesting that strategies aimed at restoring miR-181b expression may reduce the inflammatory cascade. ${ }^{44}$ Moreover, in ECs, NF-KB itself mediates the expression of the aforementioned lncRNA ANRIL, ${ }^{45}$ further indicating that non-coding RNAs could be novel targets to treat inflammatory pathway.

MiR-181a has also proven to regulate vascular inflammation, by targeting arterial SMCs. ${ }^{46}$ SMCs play a crucial role in pathological remodelling of the arterial wall. In the atherosclerotic plaque, SMCs can acquire bone-like features in response to the vasoconstricting peptide angiotensin II (AngII). AngII is produced by the ECs in close proximity, which increases expression of osteopontin, a non-collagenous protein present in the bone matrix. Interestingly, one major inducer is again a non-coding RNA, miR-181a, which orchestrates the AngII-mediated effect on osteopontin expression. $^{47}$

Transfer of miRNAs via extracellular vesicles is not exclusively found in macrophages to promote propagation of atherosclerosis and plaque instability. Also, ECs are able to communicate with other cell sub-sets in the vessel wall via apoptotic bodies, which induce a rescue mechanism that involves the C-X-C motif chemokine ligand 12 (CXCL12). ${ }^{24}$ CXCL12 is known to play a key role in tissue repair as well as angiogenesis, and can limit apoptosis. ${ }^{24}$ miR-126 has been identified to mediate CXCL12 expression in apoptotic bodiesreceiving cells. ${ }^{24}$ miR-126 regulates expression of a G-protein coupled receptor inhibitor, which then enables $\mathrm{C}-\mathrm{X}-\mathrm{C}$ motif chemokine receptor 4 to induce a feedback loop that can further increase the CXCL12 content. ${ }^{48}$

MiRNA-126 does not only play an important role in inhibiting inflammation of the vessel wall by being engulfed in apoptotic bodies released by ECs but also in ECs themselves. ${ }^{49}$ It has been shown to be expressed by ECs to inhibit vascular cell adhesion molecule 1 (VCAM-1) expression, a surface molecule found on activated ECs exclusively. ${ }^{49}$ VCAM-1 mediates adhesion of monocytes, which will then eventually migrate to the lesion site where they contribute to a potentially instable plaque. ${ }^{49}$ However, miR-126 is a prominent example for miRNAs that acts strand-specifically. MiR-126-5p rather than miR-126-3p has been found to play a beneficial role in EC proliferation, reducing atherosclerotic lesion formation. 50

Another miRNA that is used by ECs to prevent monocyte attachment and activation is miR-10a. Engulfed in extracellular vesicles, it is transferred to circulating monocytes. ${ }^{51}$ By targeting IL-1 receptor associated kinase 4 (IRAK4), a com- ponent of the NF-KB pathway in monocytes that leads to activation, the inflammatory pathway can be inhibited. Initiation and progression of atherosclerosis can thus be dampened. $^{51}$

Another example of an intercellular communication cascade is provided by the retinal non-coding RNA3 (nncRNCR3), which exerts an atheroprotective role, and gets transferred via exosomes from ECs to SMCs. LncRNCR3 confers protection against hypercholesterolaemia-induced EC and SMC dysfunction. 52

In advanced atherosclerotic lesions, many different cells types are confined in the same active area (atherosclerotic plaque). These different and distinct cell sub-types can communicate and influence each other as shown by the examples provided above. If EC dysfunction plays an initiating role in the atherosclerosis cascade, by allowing monocytes to infiltrate and initiate inflammation, in more advanced stages, SMCs become crucially relevant to determine the lesion fate. ${ }^{53}$

SMCs can receive signals that lead to trans-differentiation towards calcification, or de-differentiation towards a more proliferative phenotype, or even as more recently described transformation towards a more macrophage-like phenotype. ${ }^{54,55}$ This transformation induces a significant acceleration in phagocytic capacity, which ultimately enforces different characteristics within atherosclerotic lesions (from a more stable lesion with proliferative SMCs that produce ECM towards a more unstable phenotype with inflamed and apoptotic SMCs).

All the described events can be regulated by non-coding RNAs, offering tremendous potential as intercellular effectors and communicators. Identifying and understanding the mechanism of action of those powerful mediators will provide new ways to intervene, by operating at the cellular level and modifying the cells responsible for stabilising already formed atherosclerotic lesions (-Fig. 1).

\section{Clinical Perspective and Outlook on the Therapeutic Potential of Non-Coding RNAs}

The Cantos trial ${ }^{56}$ has proved that targeting inflammation with canakinumab (monoclonal antibody directed against IL-1 $\beta$ ) results in better outcome as compared with placebo among patients with a history of myocardial infarction and elevated high-sensitivity C-reactive protein. These results have truly opened up the 'anti-inflammatory route' for treating advanced vascular lesions. In this light, non-coding RNAs capable of modulating inflammatory networks, such as the IL-1 $\beta$-pathway, could represent interesting and therapeutically novel strategies to treat atherosclerosis. There are two types of modulations used in RNA research to interfere with miRNA or IncRNA expression: one way is to inhibit miRNAs or lncRNAs by anti-sense oligonucleotides (antimiRs, Gapmers) or by genetic knockout models (murine, and more recently porcine). The other way is to synthetically over-express the miRNA by miRNA mimics or viral vectors. Off-target effects, toxicity, easy delivery and long-term usage are obstacles to be carefully considered in this context. The 


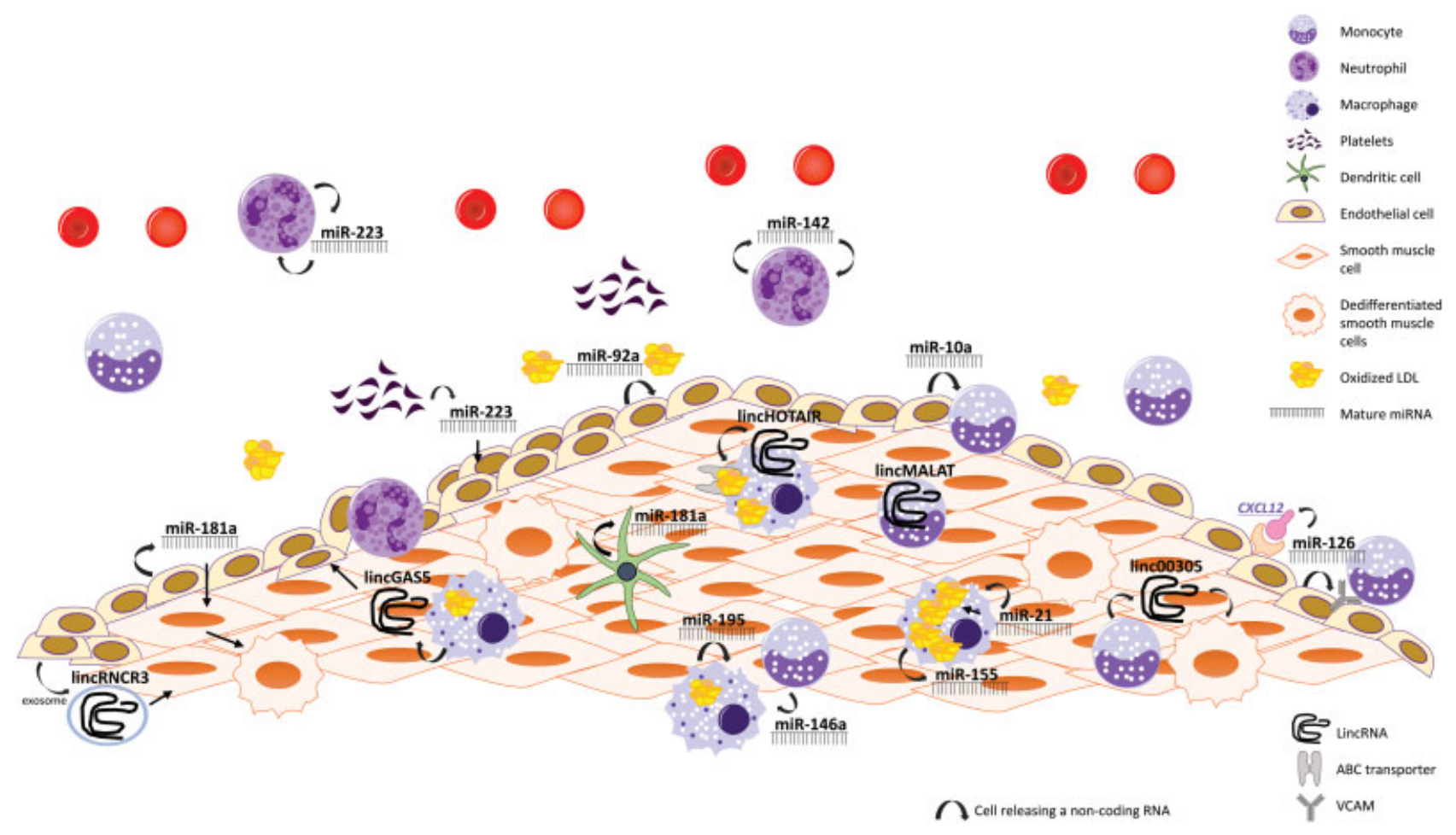

Fig. 1 Myeloid and vascular-borne non-coding RNAs aggravate vascular inflammation.

aforementioned anti-miR-92a appears to be the frontrunner in non-coding RNA-based therapies for ischaemic cardiovascular diseases. A first in-patient trial is expected to be initiated in 2019.

\section{Conflict of Interest}

None declared.

\section{Acknowledgements}

O.S. and L.M. are principal investigators in a German Research Council (DFG)-sponsored Collaborative Research Center (CRC, 1123) on 'Atherosclerosis-Mechanisms and Networks of Novel Therapeutic Targets'.

\section{References}

1 Drechsler M, Megens RT, van Zandvoort M, Weber C, Soehnlein O. Hyperlipidemia-triggered neutrophilia promotes early atherosclerosis. Circulation 2010;122(18):1837-1845

2 Ionita MG, van den Borne P, Catanzariti LM, et al. High neutrophil numbers in human carotid atherosclerotic plaques are associated with characteristics of rupture-prone lesions. Arterioscler Thromb Vasc Biol 2010;30(09):1842-1848

3 Soehnlein O, Wantha S, Simsekyilmaz S, et al. Neutrophil-derived cathelicidin protects from neointimal hyperplasia. Sci Transl Med 2011;3(103):103ra98

4 Horckmans M, Ring L, Duchene J, et al. Neutrophils orchestrate postmyocardial infarction healing by polarizing macrophages towards a reparative phenotype. Eur Heart J 2017;38(03):187-197

5 Maegdefessel L, Dalman RL, Tsao PS. Pathogenesis of abdominal aortic aneurysms: microRNAs, proteases, genetic associations. Annu Rev Med 2014;65:49-62
6 Beermann J, Piccoli MT, Viereck J, Thum T. Non-coding RNAs in development and disease: background, mechanisms, and therapeutic approaches. Physiol Rev 2016;96(04):1297-1325

7 Uchida S, Dimmeler S. Long noncoding RNAs in cardiovascular diseases. Circ Res 2015;116(04):737-750

8 Holdt LM, Teupser D. Long noncoding RNA ANRIL: Inc-ing genetic variation at the chromosome 9p21 locus to molecular mechanisms of atherosclerosis. Front Cardiovasc Med 2018;5:145

9 Holdt LM, Beutner F, Scholz M, et al. ANRIL expression is associated with atherosclerosis risk at chromosome 9p21. Arterioscler Thromb Vasc Biol 2010;30(03):620-627

10 Congrains A, Kamide K, Oguro R, et al. Genetic variants at the 9p21 locus contribute to atherosclerosis through modulation of ANRIL and CDKN2A/B. Atherosclerosis 2012;220(02):449-455

11 Arslan S, Berkan Ö, Lalem T, et al; Cardiolinc ${ }^{\mathrm{TM}}$ network. Long noncoding RNAs in the atherosclerotic plaque. Atherosclerosis 2017; 266:176-181

12 Canfrán-Duque A, Rotllan N, Zhang X, et al. Macrophage deficiency of miR-21 promotes apoptosis, plaque necrosis, and vascular inflammation during atherogenesis. EMBO Mol Med 2017;9 (09):1244-1262

13 Li K, Ching D, Luk FS, Raffai RL. Apolipoprotein E enhances microRNA-146a in monocytes and macrophages to suppress nuclear factor-kB-driven inflammation and atherosclerosis. Circ Res 2015;117(01):e1-e11

14 Cheng HS, Besla R, Li A, et al. Paradoxical suppression of atherosclerosis in the absence of microRNA-146a. Circ Res 2017;121 (04):354-367

15 Nguyen MA, Karunakaran D, Geoffrion M, et al. Extracellular vesicles secreted by atherogenic macrophages transfer microRNA to inhibit cell migration. Arterioscler Thromb Vasc Biol 2018;38(01):49-63

16 Wei Y, Zhu M, Corbalán-Campos J, Heyll K, Weber C, Schober A. Regulation of Csf1r and Bcl6 in macrophages mediates the stagespecific effects of microRNA-155 on atherosclerosis. Arterioscler Thromb Vasc Biol 2015;35(04):796-803 
17 Loyer X, Potteaux S, Vion AC, et al. Inhibition of microRNA-92a prevents endothelial dysfunction and atherosclerosis in mice. Circ Res 2014;114(03):434-443

$18 \mathrm{Wu} \mathrm{W}$, Xiao H, Laguna-Fernandez A, et al. Flow-dependent regulation of Kruppel-like factor 2 is mediated by microRNA92a. Circulation 2011;124(05):633-641

19 Fang Y, Davies PF. Site-specific microRNA-92a regulation of Kruppel-like factors 4 and 2 in atherosusceptible endothelium. Arterioscler Thromb Vasc Biol 2012;32(04):979-987

20 Bonauer A, Carmona G, Iwasaki M, et al. MicroRNA-92a controls angiogenesis and functional recovery of ischemic tissues in mice. Science 2009;324(5935):1710-1713

21 Rogg EM, Abplanalp WT, Bischof C, et al. Analysis of cell typespecific effects of microRNA-92a provides novel insights into target regulation and mechanism of action. Circulation 2018; 138(22):2545-2558

22 Sallam T, Jones M, Thomas BJ, et al. Transcriptional regulation of macrophage cholesterol efflux and atherogenesis by a long noncoding RNA. Nat Med 2018;24(03):304-312

23 Liu J, Huang GQ Ke ZP. Silence of long intergenic noncoding RNA HOTAIR ameliorates oxidative stress and inflammation response in ox-LDL-treated human macrophages by upregulating miR330-5p. J Cell Physiol 2019;234(04):5134-5142

24 Gast M, Rauch BH, Nakagawa S, et al. Immune system-mediated atherosclerosis caused by deficiency of long noncoding RNA MALAT1 in ApoE-/ - mice. Cardiovasc Res 2019;115(02):302-314

25 Chinetti-Gbaguidi G, Colin S, Staels B. Macrophage subsets in atherosclerosis. Nat Rev Cardiol 2015;12(01):10-17

26 Bras JP, Silva AM, Calin GA, Barbosa MA, Santos SG, Almeida MI. miR-195 inhibits macrophages pro-inflammatory profile and impacts the crosstalk with smooth muscle cells. PLoS One 2017;12(11):e0188530

27 Zhang DD, Wang WT, Xiong J, et al. Long noncoding RNA LINC00305 promotes inflammation by activating the AHRR-NFKB pathway in human monocytes. Sci Rep 2017;7:46204

28 Zhang BY, Jin Z, Zhao Z. Long intergenic noncoding RNA 00305 sponges miR-136 to regulate the hypoxia induced apoptosis of vascular endothelial cells. Biomed Pharmacother 2017; 94:238-243

29 Chistiakov DA, Sobenin IA, Orekhov AN, Bobryshev YV. Myeloid dendritic cells: Development, functions, and role in atherosclerotic inflammation. Immunobiology 2015;220(06):833-844

30 Subramanian M, Tabas I. Dendritic cells in atherosclerosis. Semin Immunopathol 2014;36(01):93-102

31 Wu C, Gong Y, Yuan J, et al; KaiHu. microRNA-181a represses oxLDL-stimulated inflammatory response in dendritic cell by targeting c-Fos. J Lipid Res 2012;53(11):2355-2363

32 Alemdehy MF, van Boxtel NG, de Looper HW, et al. Dicer1 deletion in myeloid-committed progenitors causes neutrophil dysplasia and blocks macrophage/dendritic cell development in mice. Blood 2012;119(20):4723-4730

33 Gantier MP. The not-so-neutral role of microRNAs in neutrophil biology. J Leukoc Biol 2013;94(04):575-583

34 Gurol T, Zhou W, Deng Q. MicroRNAs in neutrophils: potential next generation therapeutics for inflammatory ailments. Immunol Rev 2016;273(01):29-47

35 Chen CZ, Li L, Lodish HF, Bartel DP. MicroRNAs modulate hematopoietic lineage differentiation. Science 2004;303(5654):83-86

36 Naqvi AR, Fordham JB, Nares S. miR-24, miR-30b, and miR-142-3p regulate phagocytosis in myeloid inflammatory cells. J Immunol 2015;194(04):1916-1927
37 Fan HB, Liu YJ, Wang L, et al. miR-142-3p acts as an essential modulator of neutrophil development in zebrafish. Blood 2014; 124(08):1320-1330

38 Johnnidis JB, Harris MH, Wheeler RT, et al. Regulation of progenitor cell proliferation and granulocyte function by microRNA223. Nature 2008;451(7182):1125-1129

39 Bauernfeind F, Rieger A, Schildberg FA, Knolle PA, Schmid-Burgk JL, Hornung V. NLRP3 inflammasome activity is negatively controlled by miR-223. J Immunol 2012;189(08):4175-4181

40 De Jong OG, Van Balkom BW, Schiffelers RM, Bouten CV, Verhaar MC. Extracellular vesicles: potential roles in regenerative medicine. Front Immunol 2014;5:608

41 Pan $\mathrm{Y}$, Liang $\mathrm{H}$, Liu $\mathrm{H}$, et al. Platelet-secreted microRNA-223 promotes endothelial cell apoptosis induced by advanced glycation end products via targeting the insulin-like growth factor 1 receptor. J Immunol 2014;192(01):437-446

42 Neudecker V, Brodsky KS, Clambey ET, et al. Neutrophil transfer of $m i R-223$ to lung epithelial cells dampens acute lung injury in mice. Sci Transl Med 2017;9(408):eaah5360

43 Chen L, Yang W, Guo Y, et al. Exosomal IncRNA GAS5 regulates the apoptosis of macrophages and vascular endothelial cells in atherosclerosis. PLoS One 2017;12(09):e0185406

44 Sun X, Icli B, Wara AK, et al; MICU Registry. MicroRNA-181b regulates NF-кB-mediated vascular inflammation. J Clin Invest 2012;122(06):1973-1990

45 Zhou X, Han X, Wittfeldt A, et al. Long non-coding RNA ANRIL regulates inflammatory responses as a novel component of NF-KB pathway. RNA Biol 2016;13(01):98-108

46 Sun X, Sit A, Feinberg MW. Role of miR-181 family in regulating vascular inflammation and immunity. Trends Cardiovasc Med 2014;24(03):105-112

47 Remus EW, Lyle AN, Weiss D, et al. miR181a protects against angiotensin II-induced osteopontin expression in vascular smooth muscle cells. Atherosclerosis 2013;228(01):168-174

48 Zernecke A, Bidzhekov K, Noels H, et al. Delivery of microRNA-126 by apoptotic bodies induces CXCL12-dependent vascular protection. Sci Signal 2009;2(100):ra81

49 Harris TA, Yamakuchi M, Ferlito M, Mendell JT, Lowenstein CJ. MicroRNA-126 regulates endothelial expression of vascular cell adhesion molecule 1. Proc Natl Acad Sci U S A 2008;105(05):1516-1521

50 Schober A, Nazari-Jahantigh M, Wei Y, et al. MicroRNA-126-5p promotes endothelial proliferation and limits atherosclerosis by suppressing Dlk1. Nat Med 2014;20(04):368-376

51 Njock MS, Cheng HS, Dang LT, et al. Endothelial cells suppress monocyte activation through secretion of extracellular vesicles containing antiinflammatory microRNAs. Blood 2015;125(20): 3202-3212

52 Shan $\mathrm{K}$, Jiang $\mathrm{Q}$ Wang $\mathrm{XQ}$, et al. Role of long non-coding RNARNCR3 in atherosclerosis-related vascular dysfunction. Cell Death Dis 2016;7(06):e2248

53 Allahverdian S, Chaabane C, Boukais K, Francis GA, BochatonPiallat ML. Smooth muscle cell fate and plasticity in atherosclerosis. Cardiovasc Res 2018;114(04):540-550

54 Bennett MR, Sinha S, Owens GK. Vascular smooth muscle cells in atherosclerosis. Circ Res 2016;118(04):692-702

55 Shankman LS, Gomez D, Cherepanova OA, et al. KLF4-dependent phenotypic modulation of smooth muscle cells has a key role in atherosclerotic plaque pathogenesis. Nat Med 2015;21(06):628-637

56 Ridker PM, Everett BM, Thuren T, et al; CANTOS Trial Group. Antiinflammatory therapy with canakinumab for atherosclerotic disease. N Engl J Med 2017;377(12):1119-1131 